\title{
BRIEF COMMUNICATION \\ Base resolution maps reveal the importance of 5-hydroxymethylcytosine in a human glioblastoma
}

Eun-Ang Raiber ${ }^{1}$, Dario Beraldi ${ }^{1}$, Sergio Martínez Cuesta ${ }^{1}$, Gordon R. Mclnroy ${ }^{2}$, Zoya Kingsbury ${ }^{3}$, Jennifer Becq ${ }^{3}$, Terena James ${ }^{3}$, Margarida Lopes ${ }^{3}$, Kieren Allinson ${ }^{4}$, Sarah Field ${ }^{1}$, Sean Humphray ${ }^{3}$, Thomas Santarius ${ }^{5}$, Colin Watts ${ }^{5}$, David Bentley ${ }^{3}$ and Shankar Balasubramanian ${ }^{1,2,6}$

Aberrant genetic and epigenetic variations drive malignant transformation and are hallmarks of cancer. Using PCR-free sample preparation we achieved the first in-depth whole genome (hydroxyl)-methylcytosine, single-base-resolution maps from a glioblastoma tumour/margin sample of a patient. Our data provide new insights into how genetic and epigenetic variations are interrelated. In the tumour, global hypermethylation with a depletion of 5-hydroxymethylcytosine was observed. The majority of single nucleotide variations were identified as cytosine-to-thymine deamination products within CpG context, where cytosine was preferentially methylated in the margin. Notably, we observe that cells neighbouring tumour cells display epigenetic alterations characteristic of the tumour itself although genetically they appear "normal". This shows the potential transfer of epigenetic information between cells that contributes to the intratumour heterogeneity of glioblastoma. Together, our reference (epi)-genome provides a human model system for future studies that aim to explore the link between genetic and epigenetic variations in cancer progression.

npj Genomic Medicine (2017)2:6 ; doi:10.1038/s41525-017-0007-6

\section{INTRODUCTION}

Genetic and epigenetic alterations to the genome shape the development of human malignancies. The patterns of the DNA methylation mark 5-methylcytosine $(5 \mathrm{mC})$ become aberrant in human malignancies and affect cellular functions. ${ }^{1}$ The recently re-discovered DNA mark 5-hydroxymethylcytosine $(5 \mathrm{hmC})^{2,3}$ is a functionally important DNA modification, and is an intermediate in the process of active demethylation of $5 \mathrm{mC}$. In cancers, $5 \mathrm{hmC}$ patterns undergo considerable changes ${ }^{4}$ that have been linked to genome instability ${ }^{5,6}$ and remodelling of the DNA methylation pattern. ${ }^{7}$ Previous studies revealed that $5 \mathrm{hmC}$ is consistently found at significantly reduced levels in various solid tumours. ${ }^{8-10}$ Indeed, epigenetic regulators such as DNA methyltransferases (DNMT), ten-eleven-translocation (TET) proteins or isocitrate dehydrogenases (IDH), are crucial for normal and malignant cellular developement. ${ }^{11}$ Very few studies however, have effectively mapped the distribution of $5 \mathrm{hmC}$ in normal or cancer tissues. Herein, we present the first single base resolution maps of whole genomes, methylomes, and hydroxymethylomes for matched human glioblastoma and tumour margin samples.

\section{RESULTS}

Enhanced (hydroxyl)-methylome sequencing reveals global hypermethylation in tumour with loss of $5 \mathrm{hmC}$

We performed whole genome sequencing at $100 \times$ coverage of blood, tumour, and margin samples from a glioblastoma patient
(Fig. 1a) using a PCR-free library preparation. ${ }^{12}$ Total RNA sequencing of all three samples was also performed. We employed oxidative bisulfite sequencing (oxBS-seq) ${ }^{13}$ and bisulfite sequencing (BS-seq) to generate high-depth (80x) sequence coverage and built single-base resolution maps that distinguished $5 \mathrm{mC}$ and $5 \mathrm{hmC}$ modifications (Fig. $1 \mathrm{~b}$ and Supplementary Tables 1 and 2). In the margin sample, we found levels of $50 \%$ for $5 \mathrm{mC}$ and $20 \%$ for $5 \mathrm{hmC}$ integrated over all CpGs in the genome, whereas in the tumour, we observed global hypermethylation, with average levels of $60 \% 5 \mathrm{mC}$ and a drastic loss of $5 \mathrm{hmC}$ to $1.6 \%$ (Fig. 1c).

We then assessed specific regions within the genome that were differentially methylated between tumour and margin and identified substantial hypermethylation in the $3^{\prime}$-UTRs, CpGi shelves, exons and introns of the tumour compared to the margin (Fig. 1d). We observed that CpGs with high levels of $5 \mathrm{hmC}$ in margin tissue have correspondingly higher levels of $5 \mathrm{mC}$ in tumour (Fig. 1e) suggesting that elevated $5 \mathrm{mC}$ sites in tumour DNA have arisen through failure to oxidise $5 \mathrm{mC}$ to $5 \mathrm{hmC}$.

Gene promoter (hydroxyl)-methylation and transcript levels To investigate the possible mechanism that could account for the loss of $5 \mathrm{hmC}$, we looked for loss-of-function mutations or epimutations and identified single base resolution (hydroxy)methylation changes in the promoter in connection to transcript levels of key epigenetic regulators (Fig. 2a). Apparent loss of $5 \mathrm{hmC}$ in tumours can occur through loss-of-function mutations in TET

\footnotetext{
${ }^{1}$ Cancer Research UK Cambridge Institute, University of Cambridge, Cambridge, UK; ${ }^{2}$ Department of Chemistry, University of Cambridge, Cambridge, UK; ${ }^{3}$ Illumina Ltd., Chesterford Research Park, Little Chesterford, Saffron Walden, UK; ${ }^{4}$ Department of Pathology, Addenbrooke's Hospital, Cambridge University Hospitals, Cambridge, UK; ${ }^{5}$ Department of Clinical Neurosciences, Division of Neurosurgery, University of Cambridge, Cambridge, UK and ${ }^{6}$ School of Clinical Medicine, University of Cambridge, Cambridge, UK

Correspondence: Shankar Balasubramanian (sb10031@cam.ac.uk)

Eun-Ang Raiber, Dario Beraldi and Sergio Martínez Cuesta have contributed equally to this work
}

Received: 7 October 2016 Revised: 9 December 2016 Accepted: 16 December 2016

Published online: 13 March 2017 
a
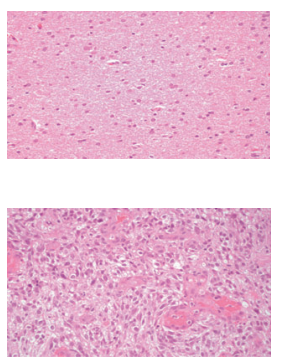

C

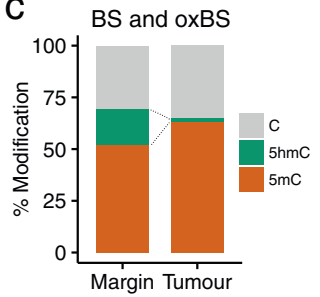

BS

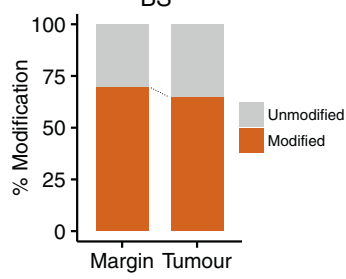

b
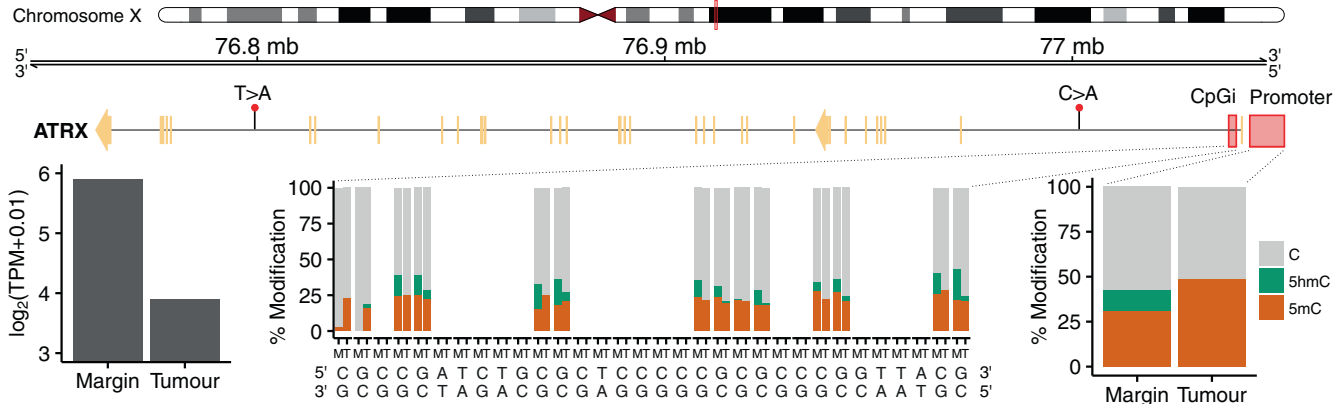

d

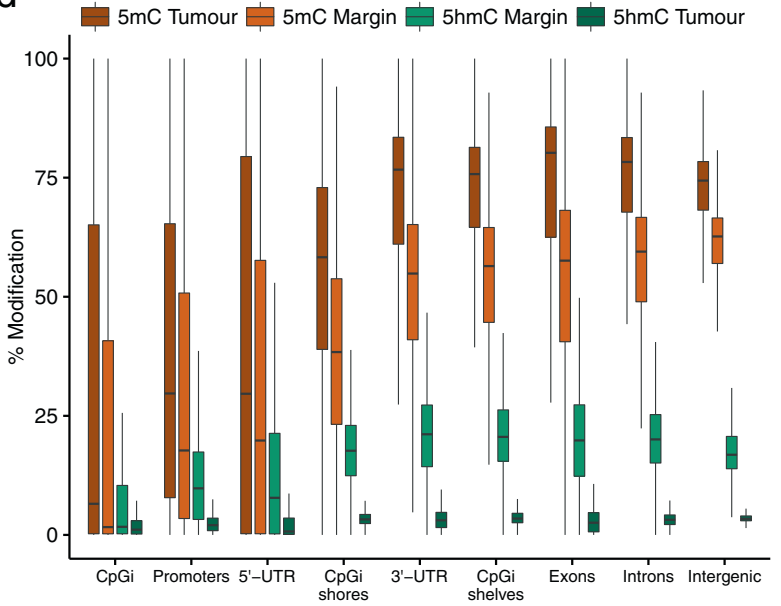

e
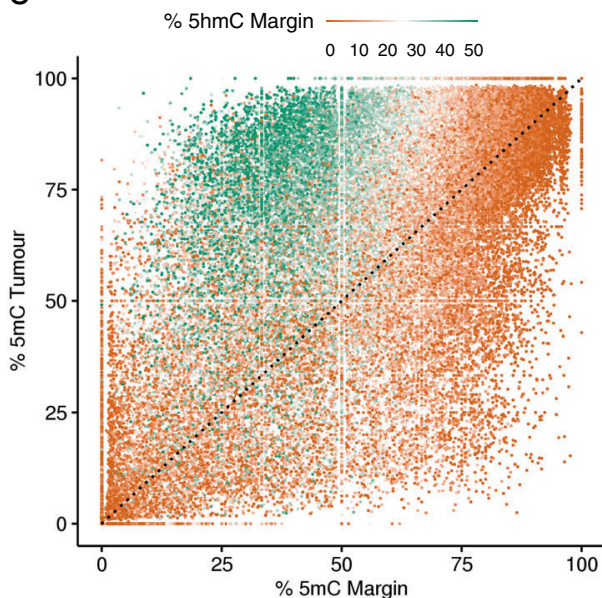

Fig. 1 Cytosine modification landscape of all CpG sites $\left(n=2.7 \times 10^{7}\right)$ in a glioblastoma patient. a Hematoxylin and eosin (H\&E) staining of the margin (top) and tumour (bottom) samples (see Methods). b Base resolution outlook of $5 \mathrm{mC}$ and $5 \mathrm{hmC}$ levels, nucleotide alterations and changes in transcript levels between margin (M) and tumour (T) in the ATRX gene. The close view highlights $32 \mathrm{bp}$ of the CpG island (chrX:77041003-77041725) located in the 5'-UTR region (middle) and average levels of modification across the $1 \mathrm{~kb}$ promoter (chrX:7704171977042719) (right). Transcript levels (left) are displayed in transcripts per million (TPM). c Combination of BS and oxBS-seq reveals a severe decrease of $5 \mathrm{hmC}$ in tumour accompanied by an increase of $5 \mathrm{mC}$, however BS-seq only cannot distinguish between $5 \mathrm{mC}$ and $5 \mathrm{hmC}$, and would suggest tumour hypomethylation. Distribution of $5 \mathrm{mC}$ and $5 \mathrm{hmC}$ in the margin and tumour samples $\mathbf{d}$ across genomic regions and $\mathbf{e}$ in a sample of $10^{5} \mathrm{CpG}$ sites colour-coded according to the level of $5 \mathrm{hmC}$ in the margin

enzymes that oxidise $5 \mathrm{mC}$ to $5 \mathrm{hmC}$, or inhibition of TET activity by the oncometabolite beta-hydroxyglutarate generated by mutant IDH1/2. ${ }^{14-17} \mathrm{IDH}$ mutations are mutually exclusive with mutations in $T E T$, at least in acute myeloid leukaemia. ${ }^{18} \mathrm{No} I D H$ mutations or lossof-function mutations in the TET genes were observed in the tumour DNA for this patient. However, we observed hypermethylation at TET2/3 gene promoters with concomitant loss of $5 \mathrm{hmC}$ at the same CpGs and a corresponding reduction in TET2/3 expression in the tumour (Fig. 2b). TET2 promoter methylation has previously been observed in low-grade diffuse gliomas lacking $I D H 1 / 2$ mutations and provides a third mechanism to cause loss in maintenance of $5 \mathrm{hmC}$ levels in the tumour. Previous literature has also linked reduced TET function to solid and myeloid malignancies $5,19,20$ and suggested a key role for TET in the prevention of cancer by suppressing cell invasion $^{21}$ and promoting genome integrity. ${ }^{5,} 6$ Our results in the current glioblastoma case are consistent with these ideas.

Transcript levels in margin are related to the levels of both, methylation and hydroxymethylation in promoters (Fig. 2c, d). The transcript levels are low at genes whose promoters have a high level of $5 \mathrm{mC}$ but a low level of $5 \mathrm{hmC}$ (sector 3, Fig. 2c). When $5 \mathrm{hmC}$ is high and $5 \mathrm{mC}$ is low (sector 2, Fig. 2c) transcript levels are high. This fits a model where $5 \mathrm{mC}$ marks silent promoters, while $5 \mathrm{hmC}$ and $\mathrm{C}$ mark active or poised promoters (Fig. 2d). ${ }^{22}$ Interestingly, even when $5 \mathrm{mC}$ is high and $5 \mathrm{hmC}$ levels shift from low to high (sectors 3 to 4 , Fig. 2c), the expression is increased. Quantitative analysis of mRNA levels revealed that 8141 genes
(24\%) were differentially expressed (logFC $>2$ or $\log F C<-2$ ) between the margin and tumour (Supplementary Fig 2) with Fig. 2e highlighting expression levels of the top ten most differentially hydroxymethylated promoters.

Patterns of genomic variations

We analysed the mutational landscape by identifying single nucleotide variants (SNVs) in tumour and margin using the blood sample as a reference. Although we identified 8169 SNVs in the tumour (Fig. 3a), the margin appeared genetically normal when compared to blood (Supplementary Fig. 3). About $50 \%$ of all SNVs in the tumour were $C$ to $T$ changes (or $G$ to $A$, opposite strand), most often in NpCpG contexts, corresponding to the mutational signature $1 \mathrm{~A}$ as described in Alexandrov et al. (Fig. 3b), though we did not observe any kataegis formation. ${ }^{23}$ SNVs identified within the coding region of several cancer genes suggest aberrations of the RTK-RAS-PI3K signalling (mutations in PTEN, PIK3R6, and NF1) and MYC signalling pathways (SMARCB1 and LZTR1), and have been recently described in a genomic characterisation of $I D H(+)$ glioma patients. ${ }^{24}$ Additionally, complex patterns of somatic structural variants were characteristic of the tumour (Fig. 3c). We observed many translocation and inversion events with additional copy number gains in chromosomes 7,17 , and 20, and copy number losses in chromosomes $1,6,9,10,11,18$, and 22 . Notably, the epigenetic modifiers TET1 (chromosome 10) and DNMT1/3B 
a

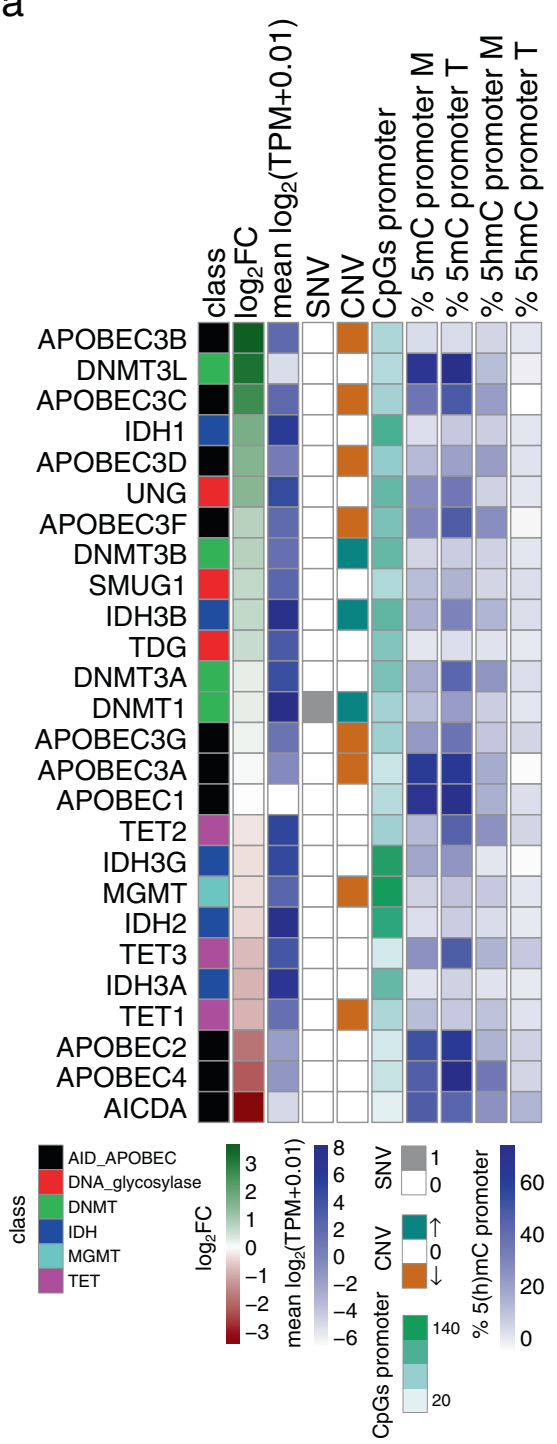

b
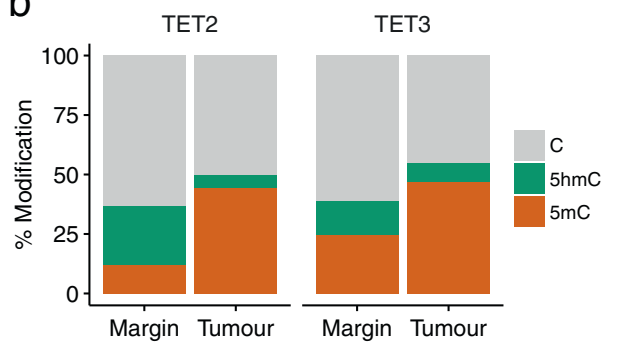

TET3

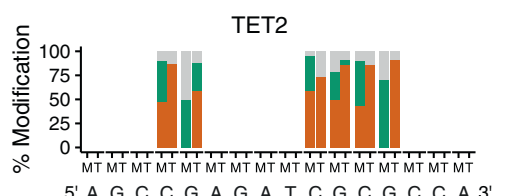

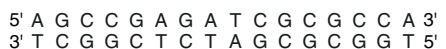
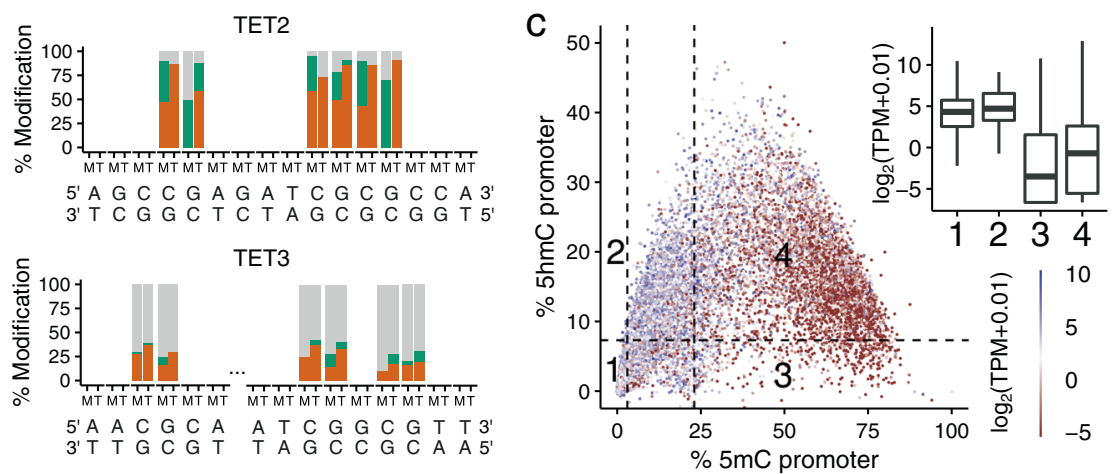

d

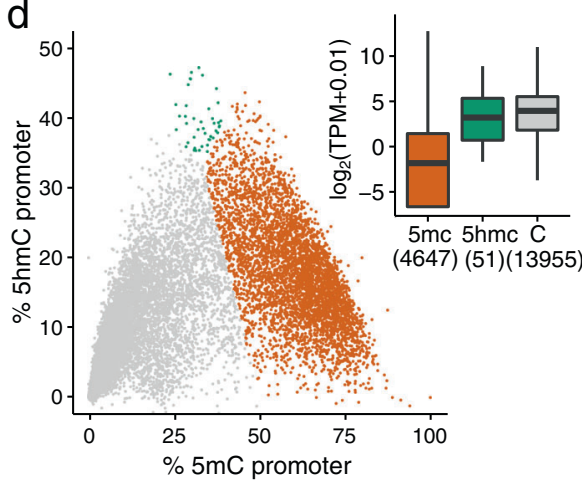

e

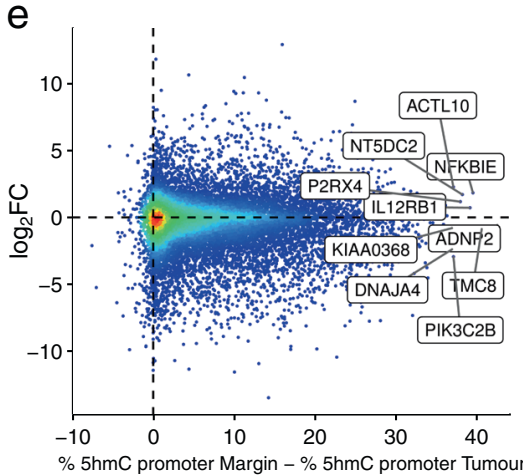

Fig. 2 Overview of the relationship between genetic changes, promoter $5 \mathrm{mC} / 5 \mathrm{hmC}$ levels and gene expression. a Summary of the molecular details of the genes involved in the turnover of cytosine modifications. Differential transcript levels between tumour and margin (log ${ }_{2} \mathrm{FC}$ where $\left.\mathrm{FC}=\left(\mathrm{TPM}_{\text {tumour }}+0.01\right) /\left(\mathrm{TPM}_{\text {margin }}+0.01\right)\right)$ and mean transcript levels $\left(\left(\log _{2}\left(\mathrm{TPM}_{\text {tumour }}+0.01\right)+\left(\log _{2}\left(\mathrm{TPM}_{\text {margin }}+0.01\right)\right) / 2\right)\right.$, $\mathrm{SNVs}(1$ : presence and 0: absence), CNVs ( $\uparrow$ : gain of copies, 0: diploid and $\downarrow$ : loss of copies and LOH: loss of heterozygosity), promoter CpG counts, and promoter $5 \mathrm{mC}$ and $5 \mathrm{hmC}$ levels (\%) in margin (M) and tumour $(\mathrm{T})$, which are colour-coded as shown in the legend. Genes bearing genomic alterations in glioma progression ${ }^{24}$ were also examined (Supplementary Fig. 1). b Average and base resolution maps of $5 \mathrm{mC}$ and $5 \mathrm{hmC}$ levels and changes in transcript abundance between margin and tumour in the promoter region ( $1 \mathrm{~kb}$ upstream of the transcription start site) of the TET2 (chr4:106066031-106067031, 22 CpG sites) and TET3 (chr2:74272449-74273449, 6 CpG sites) genes. c Cytosine modifications and changes in transcript levels across all gene promoters containing CpG sites $(n=18,653)$ in margin. Promoters are divided into four sectors according to the levels of $5 \mathrm{mC}$ and $5 \mathrm{hmC}$ : sectors $\{1,2\}$ and $\{3,4\}$ contain low and high levels of $5 \mathrm{mC}$ according to the first and third terciles $(3.2$ and $23.2 \%)$ of the $\% 5 \mathrm{mC}$ distribution respectively (horizontal axis). The median level of $5 \mathrm{hmC}(7.4 \%)$ is used to separate low and high $5 \mathrm{hmC}$ levels (vertical axis). The inset box plot displays the transcript levels for each sector. d Promoters are divided into three types depending on whether $5 \mathrm{mC}$, $5 \mathrm{hmC}$ or $\mathrm{C}$ is more abundant within the promoter. The inset box plot illustrates the transcript levels for each promoter type. e Relationship between differential transcript levels $\left(\log _{2} \mathrm{FC}\right.$ ) and differences in $5 \mathrm{hmC}$ levels between tumour and margin in gene promoters containing more than $10 \mathrm{CpG}$ sites $(n=15,716)$. The top ten promoters with larger changes in $5 \mathrm{hmC}$ levels are labelled

(chromosomes 19 and 20) were haploid and polyploid in tumour respectively, which suggests a connection between the observed hypermethylation (DNMT polyploidy) and $5 \mathrm{hmC}$ loss (diminished TET function) in the tumour.

Links between genomic and epigenomic changes

To investigate links between genetic and epigenetic mutations, we identified SNV sites resulting from the mutation of cytosine to any other base in the tumour, and studied their methylation and hydroxymethylation status in the margin (Fig. 4a). We found that SNV sites were significantly more methylated in the margin compared to non-SNV sites (Mann-Whitney test, $p$-value < 2.2e-16, two-sided). Conversely, we found that SNV sites were significantly less hydroxymethylated in the margin compared to non-SNV sites (Mann-Whitney test, $p$-value $<2.2 \mathrm{e}-16$, two-sided) with no apparent differences for all types of cytosine mutations (i.e. $C$ to, $C$ to $G$, or $C$ to A) (Fig. 4b). Regions identified to be of different ploidy in margin and tumour (Fig. 3C) did not show differences in modification levels (Fig. 4c). 


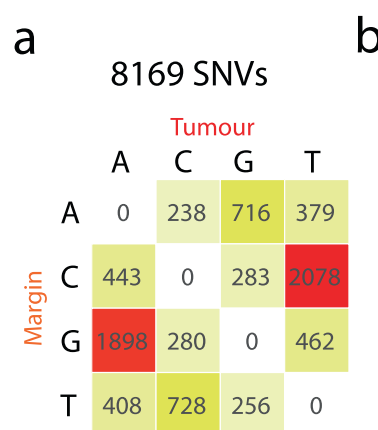

C

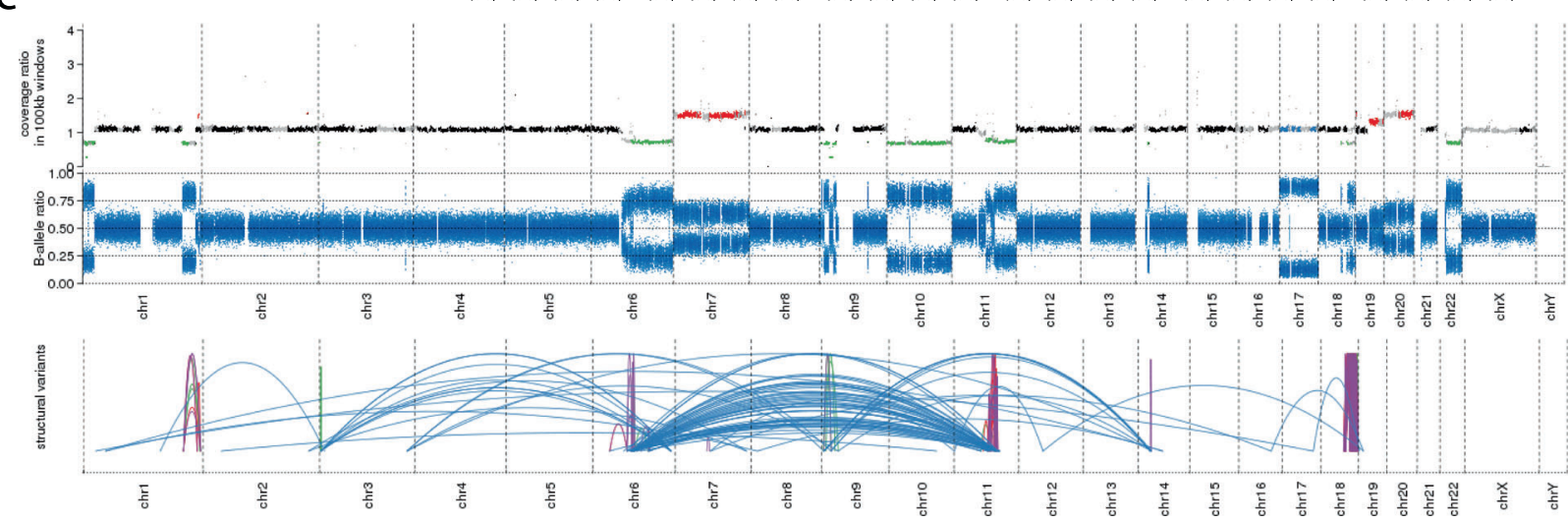

Fig. 3 Genetic variation landscape of the tumour sample. a 8169 somatic variants, mostly $C$ to $T$ transitions, identified when comparing the tumour and margin samples. b The $5^{\prime}$ and $3^{\prime}$ nucleotide context around the somatic variants in tumour suggests that most mutations occur in CpG sites. c At the top, genomic location of CNVs: polyploid regions (Gain) in red, diploid regions (Ref) in black, haploid regions (Loss) in green, and loss of heterozygosity ( $\mathrm{LOH}$ ) in blue. In the middle, B-allele ratio plot. At the bottom, genomic translocations (blue) and inversions (purple) in the tumour

Tumour purity was estimated to be $71 \%$ by genetic analysis and $60 \%$ by immunohistochemistry (see Methods). Based on tumour purity, we calculated the expected global level of $5 \mathrm{hmC}$ to have a lower limit of $7 \%$ (Table 1), assuming complete loss of $5 \mathrm{hmC}$ in the tumour. We measured actual levels of $5 \mathrm{hmC}$ in the tumour sample to be $1.6 \%$, more than four-fold lower than the estimated lower limit.

\section{DISCUSSION}

We used a PCR-free approach to generate accurate methylomes and hydroxymethylomes. Importantly, our ability to resolve $5 \mathrm{mC}$ and $5 \mathrm{hmC}$ signals reveals global hypermethylation in the tumour, contrary to the appearance of global hypomethylation when using only BS-seq, which actually measures the sum of $5 \mathrm{mC}$ and $5 \mathrm{hmC}$ and fails to resolve the two signals. Global hypermethylation has just recently also been reported for kidney cancer using an alternative method that was able to distinguish $5 \mathrm{mC}$ from $5 \mathrm{hmC}{ }^{25}$ While genome-wide hypomethylation ${ }^{26,27}$ is somewhat regarded as an epigenetic hallmark of tumorigenesis and has been implicated in the evolution of human glioblastomas, ${ }^{28}$ our results suggest that data obtained from bisulfite-only approaches need to be re-interpreted.

TET2 promoter methylation has previously been observed in low-grade diffuse gliomas lacking IDH1/2 mutations and provides a third mechanism to cause loss in maintenance of $5 \mathrm{hmC}$ levels in the tumour. Previous literature has also linked reduced TET function to solid and myeloid malignancies ${ }^{5,19,20}$ and suggested a key role for TET in the prevention of cancer by suppressing cell invasion $^{21}$ and promoting genome integrity. ${ }^{5,6}$ Our results are consistent with this mechanism in the current glioblastoma case.

$5 \mathrm{mCpG}$ sequences have previously been reported to be mutational hotspots in human genetic disease and cancer- relevant genes, probably through the deamination of $5 \mathrm{mC}$ to $\mathrm{T}^{29}$ Our data show that $5 \mathrm{mCpG}$ sites are predisposed to mutations, whereas $5 \mathrm{hmCpG}$ sites are protected from mutations during tumorigenesis, mirroring observations recently reported for brain and kidney cancer, and myeloid leukaemias. ${ }^{30}$ Interestingly, the 'protection' at $5 \mathrm{hmCpG}$ sites holds for all types of cytosine mutations ( $C$ to $T, C$ to $G$, or $C$ to $A$ ).

Our observation that $5 \mathrm{hmC}$ levels in the tumour are significantly lower than the estimated lower limit suggests a loss of $5 \mathrm{hmC}$ in the genetically "normal" cells within the tumour mass. Earlier studies reported that genome-wide methylation changes in tumours can be acquired in adjacent normal cells linking $5 \mathrm{mC}$ to field defects. ${ }^{31-33}$ The concept of field defects ${ }^{34}$ has been used to describe the early events in the stepwise transformation of the cancer that can potentially lead to further oncogenic changes. To the best of our knowledge, this is the first observation of acquired, early changes in $5 \mathrm{hmC}$ in proximal cells.

Accurate, high resolution whole genome maps have allowed us to discern a role for $5 \mathrm{hmC}$ in protecting the genome against somatic mutations, observe global hypermethylation in a tumour and provide new evidence that associates $5 \mathrm{hmC}$ with epigenetic transformation in genetically normal cells adjacent to the tumour. Epigenetic analysis of $5 \mathrm{hmC}$, in addition to $5 \mathrm{mC}$, may foster diagnostic approaches in the future.

\section{METHODS}

Clinical events

A 71-year old woman underwent supra-total resection of a right frontal intrinsic tumour with maximum diameter of the enhancing component of $19 \mathrm{~mm}$, surrounded by only minimal oedema. Histological examination confirmed the diagnosis of glioblastoma 
a

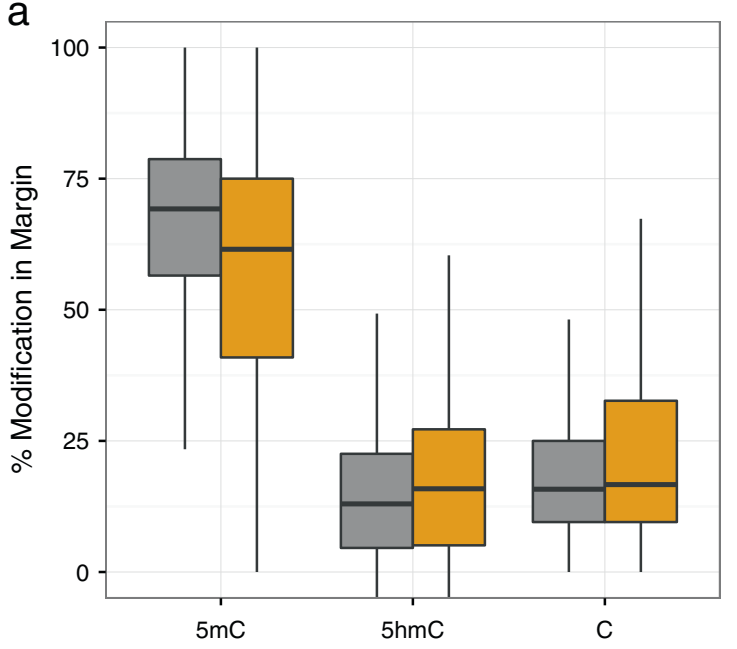

SNV n=2610 ש non-SNV n=54479299 b

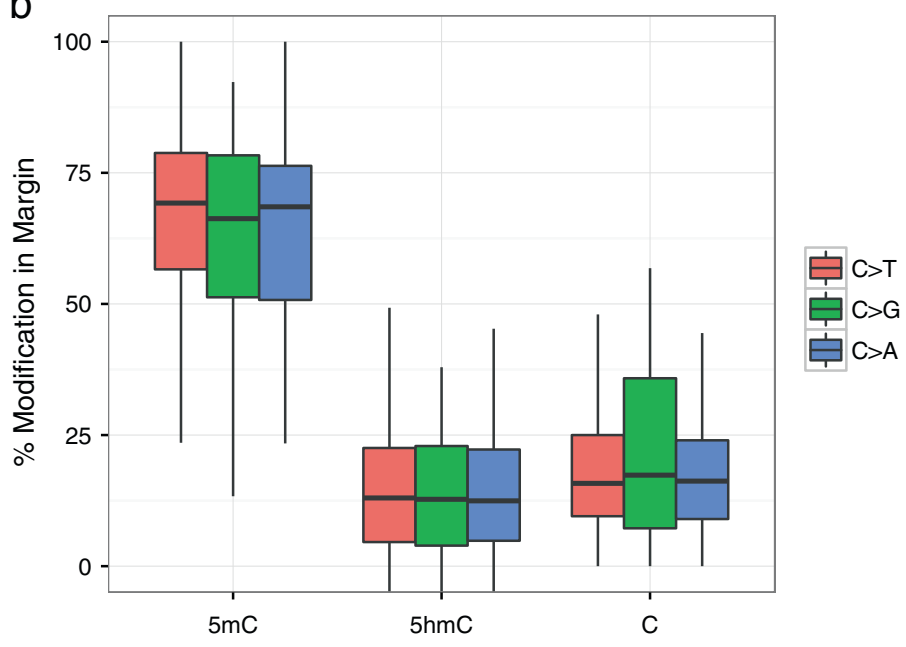

C

Margin

Tumour

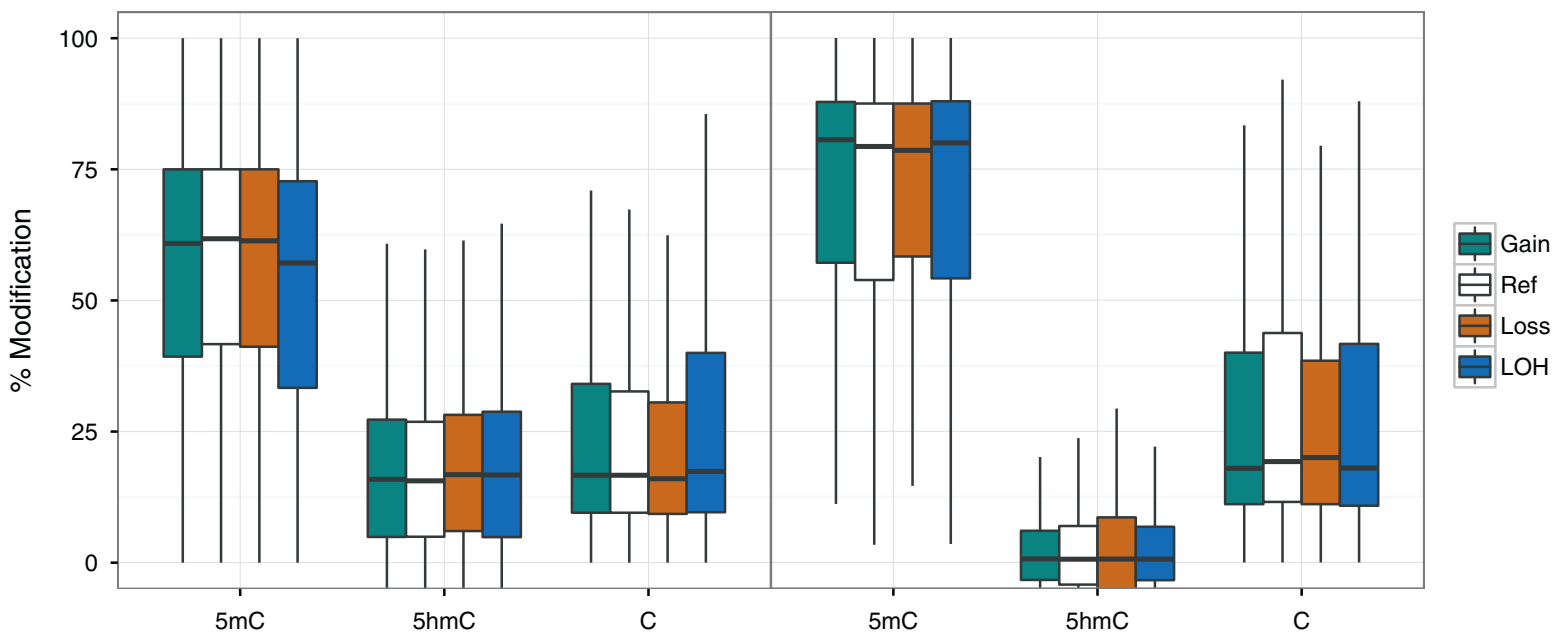

Fig. 4 Link between genetic variation and cytosine modifications. a Analysis of $5 \mathrm{mC}$ and $5 \mathrm{hmC}$ levels within the margin $\mathrm{CpG}$ sites that are mutated in tumour (SNVs). b Differences in modification levels depending on the mutated base in tumour: C>T $(n=2527), C>G(n=32)$ and C>A $(n=51)$. c Distribution of modification levels in margin and tumour in regions with gain or loss of copies, diploid (Ref) or loss of heterozygosity (LOH) according to the CNV analysis of the tumour sample (Fig. 3c)

Table 1. Overall cytosine modification levels of all CpG sites $(n=2.7 \times$ $10^{7}$ ) in a glioblastoma patient

\begin{tabular}{llc|}
\hline Sample & $\% 5 \mathrm{mC}$ & $\% 5 \mathrm{hmC}$ \\
\hline Margin & 52.1 & 17.5 \\
Tumour & 63.5 & 1.6 \\
\hline
\end{tabular}

multiforme (WHO 9440/3). Tissue collection protocols were compliant with the UK Human Tissue Act 2004 (HTA License ref. 12315) and approved by the Local Regional Ethics Committee (LREC ref. 04/Q0108/60). Informed consent was obtained from the patient before surgery. Surgical samples were taken from the enhancing tumour mass and corresponding non-enhancing margin using advanced surgical techniques described previously. ${ }^{35}$ After rapid recovery she underwent six weeks of radiotherapy (60Gy in 30 fractions) with concurrent temozolomide (TMZ) treatment. She then completed two cycles of adjuvant TMZ treatment during 12 weeks. This was stopped because of poor tolerance. While her 9-months post-operative scan was clear of tumour, her 11-months check demonstrated tumour recurrence and she died 3 months later from the combination of tumour progression and thromboembolic complications.

\section{Samples}

Brain tumour biopsies were fixed in 10\% formalin and embedded in paraffin wax from which 4- $\mu$ m-thick sections were cut and stained with haematoxylin and eosin (H\&E). The diagnosis of glioblastoma multiforme (WHO 9440/3) was made by light microscopical examination of H\&E-stained sections. Glioblastoma was defined by WHO-2016 criteria of an infiltrative astrocytoma with proliferative activity, necrosis and/or microvascular proliferation.

To determine the IDH1 status by immunohistochemistry, the 4$\mu \mathrm{m}$-thick sections were dried at $60^{\circ} \mathrm{C}$ for $2 \mathrm{~h}$ and further processed on a Bond Max (Leica). After a 60 -min pre-treatment with cell conditioner $2(\mathrm{pH} 6)$ the slides were incubated with 1:80 diluted $\mathrm{H} 09$ anti-IDH1 R132H antibody (Dianova, Hamburg, Germany) at 
room temperature for $30 \mathrm{~min}$. A standard 3,3'-diaminobenzidine (DAB) detection kit was used for chromogenic detection. The common IDH1 mutation (R132H) was not detected.

To determine the MGMT promoter methylation, H\&E-stained slides were reviewed and neoplastic cell-rich tissue was dissected from consecutive unstained sections. DNA was extracted from the dissected tissue using the QIAamp DNA FFPE Tissue Kit (Qiagen) and was bisulphite-converted using the EpiTect Bisulphite Kit (Qiagen). The MGMT promoter methylation was determined by pyrosequencing of four CpG sites (CpGs 76-79) in exon 1 of the MGMT gene using the CE-Marked therascreen MGMT Pyro Kit on a Pyromark Q24 System (Qiagen). Significant levels of MGMT promoter methylation were not detected.

To determine the tumour cell purity of the samples, H\&Estained slides were reviewed and cell counting was performed on multiple high-powered fields. The tumour cell purity of both the tumour and its margin was assessed by a pathologist prior to molecular profiling. No tumour cells were detected in the margins and the tumour was estimated to consist of $60 \%$ tumour cells.

\section{DNA and RNA extraction for sequencing}

Each tumour and margin sample was frozen to $-80^{\circ} \mathrm{C}$ immediately on collection. The frozen samples were then defrosted and a $50 \mathrm{mg}$ portion taken for DNA/RNA extraction. The samples were homogenised and then divided for DNA and RNA extraction. DNA extraction of both tissues and blood was performed using the QIAGEN DNeasy Blood and Tissue Kit, RNA extraction was performed using the QIAGEN RNeasy Mini Kit. Quantification was performed using the Qubit quantification assay. The tumour/ normal pair used in this study was the sample judged most pure by comparison with blood DNA using whole genome sequencing.

PCR-free oxBS and BS library preparation and sequencing PCR-free ReBuilT libraries for bisulfite sequencing were generated following our previously published method. ${ }^{12}$ For the generation of PCR-free libraries for oxBS, libraries were oxidised (TrueMethyl Kit from (EGX) prior to bisulfite treatment. In brief, 350 and $175 \mathrm{ng}$ of sonicated DNA from tumour and margin respectively were end repaired and $\mathrm{dA}$-tailed before ligation of customized adapter pair. After (oxidation)-bisulfite treatment, degraded fragments were recovered through primer extension before $\mathrm{dA}$-tailing and second adapter ligation. Libraries were subsequently immobilized on streptavidin coated magnetic beads, washed with binding buffer, eluted with $50 \mathrm{mM} \mathrm{NaOH}$ at $60^{\circ} \mathrm{C}$ for $15 \mathrm{~min}$. BS and oxBS-seq libraries were sequenced on an Illumina HiSeq 2500 platform, V4 chemistry, 2x101+7 index cycles.

RNA-seq library preparation and sequencing

Libraries of the tumour and margin samples were prepared using TruSeq RNA Access kit (Illumina), using an input of 40 and $20 \mathrm{ng}$ respectively. The libraries were sequenced for $2 \times 75$ cycles on HiSeq 2500 in Rapid mode achieving an average coding coverage of 108x (F2), 136X (F3), 277x (M1), 272x (M3).

\section{Computational analysis}

Code availability. Details of the analysis are available in the manuscript's GitHub repository (https://github.com/sblabbioinformatics/epigenetics-of-glioblastoma).

DNA-seq data analysis. Libraries were sequenced on an Illumina HiSeq 2500 platform. Alignment to the human reference genome GRCh37 and quality control was performed using Isaac. $^{36}$ Identification of somatic SNVs and small somatic indels ( $<50 \mathrm{bp}$ ) was performed by Strelka. ${ }^{37}$ Large copy number variants and structural variants were respectively called with Canvas $^{38}$ and Manta. ${ }^{39}$ The full workflow can be found in the Tumour Normal application of the Illumina BaseSpace platform (http://support. illumina.com/content/dam/illumina-support/help/BaseSpace_App _TumorNormal_v2/tumor-normal-v2-help.htm).

BS and oxBS-seq data analysis. Raw reads were trimmed to remove adapter sequences ligated to the 3 '-end using cutadapt ${ }^{40}$ and aligned to the human reference genome hg19 using bwameth, ${ }^{41}$ a wrapper around bwa-mem. ${ }^{42}$

RNA-seq data analysis. As above, reads were trimmed to remove adapter sequences using cutadapt and aligned to reference transcripts obtained from Ensembl $^{43}$ and quantified using kallisto. $^{44}$

Tumour purity estimation. The copy number variant analysis using Canvas ${ }^{38}$ provided us with an estimate of $71 \%$ purity of the tumour sample. ${ }^{45}$ This software calculates coverage and tumour SNV allele frequencies at heterozygous germline positions along the genome. It then assigns copy numbers per genomic regions and infers genome-wide ploidy and purity by fitting the data to expected models for each copy number state given purity and diploid coverage level combinations. Purity is then derived from the best fitting model.

Data availability

Data are available in the ArrayExpress database (www.ebi.ac.uk/ arrayexpress) under accession number E-MTAB-5171

\section{ACKNOWLEDGEMENTS}

The Balasubramanian laboratory is supported by core funding from Cancer Research UK (C14303/A17197). S.B. is a Senior Investigator of the Wellcome Trust (grant no. $099232 / z / 12 / z)$. Colin Watts is supported by funding from CRUK and The Brain Tumour Charity.

\section{AUTHOR CONTRIBUTIONS}

All authors contributed to the conception and design of the experiments. T. S. and K. A. provided samples and clinical data. E.A.R., G.R.M., S.F., T.J., M.L. and Z.K. performed the experiments. D.B., S.M.C. and J.B. performed computational analyses. All authors analysed and interpreted the data. E.A.R., D.B., S.M.C. and S.B. wrote the manuscript, with contributions from all authors.

\section{COMPETING INTERESTS}

S.B. is a founder and shareholder of Cambridge Epigenetix Ltd. The authors otherwise declare no competing interests.

\section{REFERENCES}

1. Baylin, S. B. et al. Aberrant patterns of DNA methylation, chromatin formation and gene expression in cancer. Hum. Mol. Genet. 10, 687-692 (2001).

2. Kriaucionis, S. \& Heintz, N. The nuclear DNA base 5-hydroxymethylcytosine is present in Purkinje neurons and the brain. Science 324, 929-930 (2009).

3. Tahiliani, M. et al. Conversion of 5-methylcytosine to 5-hydroxymethylcytosine in mammalian DNA by MLL partner TET1. Science 324, 930-935 (2009).

4. Pfeifer, G. P., Kadam, S. \& Jin, S.-G. 5-Hydroxymethylcytosine and its potential roles in development and cancer. Epigenetics Chromatin 6, 10 (2013).

5. An, J. et al. Acute loss of TET function results in aggressive myeloid cancer in mice. Nat. Commun. 6, 10071 (2015).

6. Kafer, G. R. et al. 5-Hydroxymethylcytosine marks sites of DNA damage and promotes genome stability. Cell Rep. 14, 1283-1292 (2016).

7. Ficz, G. \& Gribben, J. G. Loss of 5-hydroxymethylcytosine in cancer: cause or consequence? Genomics 104, 352-357 (2014).

8. Haffner, M. C. et al. Global 5-hydroxymethylcytosine content is significantly reduced in tissue stem/progenitor cell compartments and in human cancers. Oncotarget 2, 627-637 (2011).

9. Yang et al. Tumor development is associated with decrease of TET gene expression and 5-methylcytosine hydroxylation. Oncogene 32, 663-669 (2013). 
10. Kudo et al. Loss of 5-hydroxymethylcytosine is accompanied with malignant cellular transformation. Cancer Sci. 103, 670-676 (2012).

11. Scouzic, L., Mouly, E. \& Bernard, O. A. TET proteins and the control of cytosine demethylation in cancer. Genome Med. 7, 9 (2015)

12. Mclnroy, G. R. et al. Enhanced methylation analysis by recovery of unsequenceable fragments. PLoS One 11, e0152322 (2016).

13. Booth, M. J. et al. Quantitative sequencing of 5-methylcytosine and 5hydroxymethylcytosine at single-base resolution. Science 336, 934-937 (2012).

14. Figueroa, M. E. et al. Leukemic IDH1 and IDH2 mutations result in a hypermethylation phenotype, disrupt TET2 function, and impair hematopoietic differentiation. Cancer Cell 18, 553-567 (2010).

15. Xu, W. et al. Oncometabolite 2-hydroxyglutarate is a competitive inhibitor of a ketoglutarate-dependent dioxygenases. Cancer Cell 19, 17-30 (2011).

16. Delhommeau, F. et al. Mutation in TET2 in myeloid cancers. N. Engl. J. Med. 360 , 2289-2301 (2009).

17. Mullighan, C. G. TET2 mutations in myelodysplasia and myeloid malignancies. Nat. Genet. 41, 766-767 (2009).

18. Yang, H., Ye, D., Guan, K. L. \& Xiong, Y. IDH1 and IDH2 mutations in tumorigenesis: mechanistic insights and clinical perspectives. Clin. Cancer Res. 18, 5562-5571 (2012).

19. Yang, $H$. et al. Tumor development is associated with decrease of TET gene expression and 5-methylcytosine hydroxylation. Oncogene 32, 663-669 (2012).

20. Huang, Y. \& Rao, A. Connections between TET proteins and aberrant DNA modification in cancer. Trends Genet. 30, 1-11 (2014).

21. Hsu, C. H. et al. TET1 suppresses cancer invasion by activating the tissue inhibitors of Metalloproteinases. Cell Rep. 2, 568-579 (2012).

22. Ficz, G. et al. Dynamic regulation of 5-hydroxymethylcytosine in mouse ES cells and during differentiation. Nature 473, 398-402 (2011).

23. Alexandrov, L. B. et al. Signatures of mutational processes in human cancer. Nature 500, 415-421 (2013).

24. Bai, H. et al. Integrated genomic characterization of IDH1-mutant glioma malignant progression. Nat. Genet. 48, 59-66 (2015).

25. Chen, K. et al. Loss of 5-hydroxymethylcytosine is linked to gene body hypermethylation in kidney cancer. Cell Res. 26, 103-118 (2016).

26. Gama-Sosa, M. A. et al. The 5-methylcytosine content of DNA from human tumors. Nucleic Acids Res. 11, 6883-6894 (1983).

27. Ehrlich, M. DNA methylation in cancer: too much, but also too little. Oncogene $\mathbf{2 1}$ 5400-5413 (2002).

28. Mazor, T. et al. DNA methylation and somatic mutations converge on the cell cycle and define similar evolutionary histories in brain tumors. Cancer Cell 28, 307-317 (2015).

29 Pfeifer, G. P. Mutagenesis at methylated CpG sequences. Curr. Top. Microbiol. Immunol. 301, 259-281 (2006).

30. Tomkova, M., McClellan, M., Kriaucionis, S. \& Schuster-Boeckler, B. 5hydroxymethylcytosine marks regions with reduced mutation frequency in human DNA. Elife 5, 459-466 (2016).
31. Suter, C. M., Martin, D. I. \& Ward, R. I. Hypomethylation of L1 retrotransposons in colorectal cancer and adjacent normal tissue. Int. J. Colorectal. Dis. 19, 95-101 (2004)

32. Teschendorff, A. E. et al. DNA methylation outliers in normal breast tissue identify field defects that are enriched in cancer. Nat. Commun. 7, 10478 (2016).

33. Katsurano, M. et al. Early-stage formation of an epigenetic field defect in a mouse colitis model, and non-essential roles of T- and B-cells in DNA methylation induction. Oncogene 31, 342-351 (2012).

34. Slaughter, D. P., Southwick, H. W. \& Smejkal, W. Field cancerization in oral stratified squamous epithelium; clinical implications of multicentric origin. Cancer $\mathbf{6}$ 963-968 (1953).

35. Piccirillo, S. G. M. et al. Fluorescence-guided surgical sampling of glioblastoma identifies phenotypically distinct tumour-initiating cell populations in the tumour mass and margin. Br. J. Cancer 107, 462-468 (2012).

36. Raczy, C. et al. Isaac: ultra-fast whole-genome secondary analysis on Illumina sequencing platforms. Bioinformatics 29, 2041-2043 (2013).

37. Saunders, C. T. et al. Strelka: accurate somatic small-variant calling from sequenced tumor-normal sample pairs. Bioinformatics 28, 1811-1817 (2012).

38. Roller, E., Ivakhno, S., Lee, S., Royce, T. \& Tanner, S. Canvas: versatile and scalable detection of copy number variants. Bioinformatics 32, 2375-2377 (2016).

39. Chen, X. et al. Manta: rapid detection of structural variants and indels for germline and cancer sequencing applications. Bioinformatics 32, 1220-1222 (2016).

40. Martin, M. Cutadapt removes adapter sequences from high-throughput sequencing reads. EMBnet J. 17, 10 (2011).

41. Pedersen, B. S., Eyring, K., De, S., Yang, I. V. \& Schwartz, D. A. Fast and accurate alignment of long bisulfite-seq reads. arXiv 1401.1129, 1-2 (2014).

42. $\mathrm{Li}, \mathrm{H}$. Aligning sequence reads, clone sequences and assembly contigs with BWAMEM. arXiv 1303.3997, 1-3 (2013).

43. Yates, A. et al. Ensembl 2016. Nucleic Acids Res. 44, D710-D716 (2016).

44. Bray, N. L., Pimentel, H., Melsted, P. \& Pachter, L. Near-optimal probabilistic RNAseq quantification. Nat. Biotechnol. 34, 525-527 (2016).

45. Forbes, S. A. et al. COSMIC: exploring the world's knowledge of somatic mutations in human cancer. Nucleic Acids Res. 43, D805-D811 (2015).

(i) This work is licensed under a Creative Commons Attribution 4.0 International License. The images or other third party material in this article are included in the article's Creative Commons license, unless indicated otherwise in the credit line; if the material is not included under the Creative Commons license, users will need to obtain permission from the license holder to reproduce the material. To view a copy of this license, visit http://creativecommons.org/licenses/by/ 4.0/

(c) The Author(s) 2017

Supplementary Information accompanies the paper on the npj Genomic Medicine website (doi:10.1038/s41525-017-0007-6). 